\title{
Spatial Modulation Microscopy for Real-Time Imaging of Plasmonic Nanoparticles and Cells
}

\author{
N. Fairbairn,${ }^{1}$ R. A. Light,${ }^{2}$ R. Carter,${ }^{3}$ R. Fernandes,${ }^{1}$ A. G. Kanaras,${ }^{1}$ \\ T. J. Elliott, ${ }^{3}$ M. G. Somekh, ${ }^{2}$ M. C. Pitter, ${ }^{2}$ and O. L. Muskens ${ }^{1}$ \\ ${ }^{1}$ Institute for Life Sciences and Faculty of Physical and Applied Sciences, \\ University of Southampton, Highfield, Southampton SO17, 1BJ, United Kingdom \\ ${ }^{2}$ Applied Optics Group, Faculty of Engineering, University of Nottingham, Nottingham NG7 2RD, United Kingdom. \\ ${ }^{3}$ Institute for Life Sciences and Faculty of Medicine, University of Southampton, United Kingdom
}

(Dated: March 28, 2012)

\begin{abstract}
Spatial modulation microscopy is a technique originally developed for quantitative spectroscopy of individual nano-objects. Here, a parallel implementation of the spatial modulation microscopy technique is demonstrated based on a line detector capable of demodulation at $\mathrm{kHz}$ frequencies. The capabilities of the imaging system are shown using an array of plasmonic nanoantennas and dendritic cells incubated with gold nanoparticles.
\end{abstract}

PACS numbers:

With the increasing employment of nanomaterials in physical and biomedical science, sensitive methods capable of screening and characterizing these materials are needed. Currently there is a range of optical microscopy techniques capable of imaging individual nanoparticles 3]. For nonfluorescent particles, interactions take place through absorption and/or scattering. Detection of scattered intensity, generally known as darkfield microscopy, is the most widely used technique, combining background-free imaging with a high spectral selectivity for e.g. optical sensing [1, 2]. The efficiency of light scattering is greatly reduced with decreasing particle size, proportional to the square of the particle volume, which can be overcome using more advanced interferometric detection schemes 3, 4]. Also scattering-based techniques are not suitable for materials with an a small dielectric contrast, i.e. index-matched particles in solution.

A fundamentally different method of detection relies on detection of absorption rather than scattering of light. The most sensitive of these techniques is photothermal imaging, where a thermal response caused by local absorption can be detected with single-molecule sensitivity 5]. Photothermal imaging methods require a relatively high laser intensity $\left(\mathrm{MW} / \mathrm{cm}^{2}\right)$ over a diffraction limited spot, which limits the scalability of photothermal imaging in real-time and live cell applications. Spatial modulation microscopy (SMM) has been introduced as a technique for quantitative analysis of the optical extinction cross-section of small metal nanoparticles [6, 7]. The method is based on recovery of a small modulation component in the transmitted (or reflected) light using lock-in detection. This spatial modulation is achieved by means of a periodical displacement of the specimen in a Gaussian laser focus. While the technique is less sensitive than photothermal imaging, it allows for precise in-situ quantitative spectroscopy of nanomaterials which can then be correlated to other properties such as their ultrafast response [8]. In addition, the SMM technique is scalable as it works at moderate optical intensities, i.e. a few $\mathrm{W} / \mathrm{cm}^{2}$ per pixel, and requires only a small focal width in one dimension. No reports have been made yet on the potential of SMM for real-time imaging in biological systems.

Here, we demonstrate the integration of SMM into a viable imaging system covering an area of tens of micrometers at a rate of around one image per second. The fast scanning spatial modulation method, makes use of a recently developed CMOS camera technology combining multiple wells and a phase stepping technique to detect a modulating signal [9, 10]. The line camera replaces conventional lock-in amplification to detect the modulating signal produced by the spatial modulation technique. The camera features a pixel well depth of 2 billion electrons, providing a theoretical shot noise level of $2 \times 10^{-5}$ per well. In addition, a series of four on-chip wells per pixel allows fast acquisition of four frames in each modulation period, which can be used to recover the first and second harmonic spatial modulation at $\mathrm{kHz}$ frequencies. The resulting intensities are converted into 16-bit integers, each count representing $3 \times 10^{4}$ electrons.

The experimental setup is shown in Fig. 1. A transmission microscope was used consisting of two $100 \times, 0.9$ NA microscope objectives. A vertical line focus was cre-

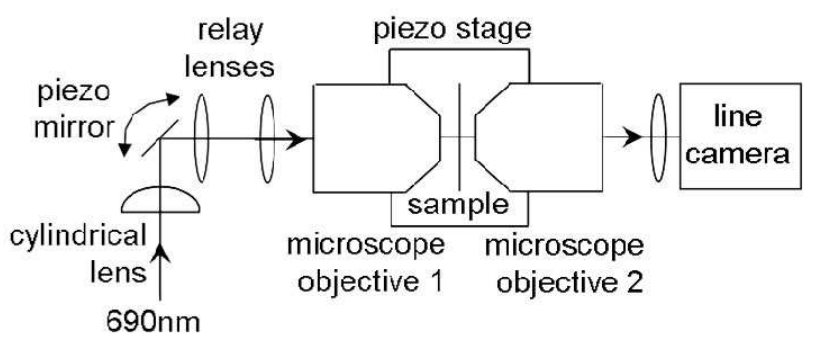

FIG. 1: Experimental setup for spatial modulation microscopy. 


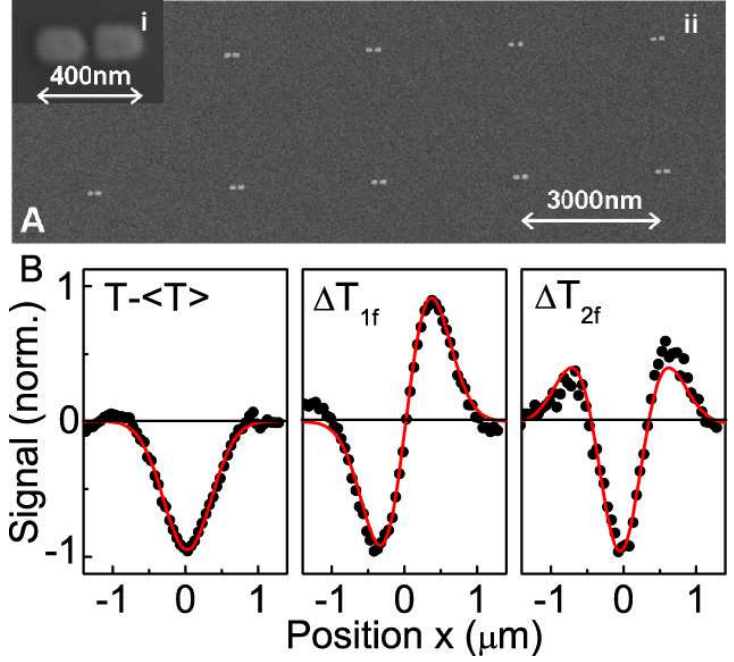

FIG. 2: a) SEM images of nanoantennnas, with close up of single antenna. (b) Experimental data for a single antenna for intensity $T-\langle T\rangle$, and SMM profiles at first $\left(\Delta T_{1 f}\right)$ and second harmonic $\left(\Delta T_{2 f}\right)$ of the modulation frequency, together with fits (lines) to the derivatives of a Gaussian beam profile of $0.8 \pm 0.01 \mu \mathrm{m}$ width.

ated from a $690 \mathrm{~nm}$ beam by imaging of the focus of a cylindrical lens onto the sample. The transmitted light was subsequently collected by the second objective and imaged onto the CMOS line array. Spatial modulation was implemented by periodically tilting of a custom-built piezo-actuated flexure mirror at a frequency of $1.3 \mathrm{kHz}$. The microscopy objective acts as a fourier element converting the angular tilt into a spatial displacement of the line focus. This method greatly improves the flexibility of the SMM method in combination with bulky or liquid specimens compared to previous implementations based on a piezoelectric sample displacement [6]. In order to optimally exploit the dynamic range of the 256-pixel array at a typical frame rate of 5200 frames per second, a total illumination power of around $2 \mathrm{~mW}$ is required. Given the optical resolution of $0.2 \mu \mathrm{m}$ per pixel this results in an average local intensity of around $10 \mathrm{~W} / \mathrm{cm}^{2}$.

The SMM imaging performance was tested by mapping an array of lithographically defined gold nanoantennas on an ITO coated glass substrate. The nanoantennas, shown in Fig. 2(a), comprise of two arms of $200 \mathrm{~nm}$ in length and $100 \mathrm{~nm}$ wide, with a thickness of $25 \mathrm{~nm}$, and were designed to have a resonant dipole mode at around $850 \mathrm{~nm}$ [8]. Two-dimensional SMM maps were made of the transmitted intensity $T$ as well as the first and second harmonic components of the spatial modulation intensity, respectively denoted as $\Delta T_{1 f}$ and $\Delta T_{2 f}[\underline{6}]$. For the intensity graph, an average line frame in absence of antennas (denoted as $\langle T\rangle$ ) was subtracted to remove a constant variation in pixel illumination. Figure2(b) shows typical cross-sections through a single antenna selected from a larger image shown in Fig. 3 The signals can be well

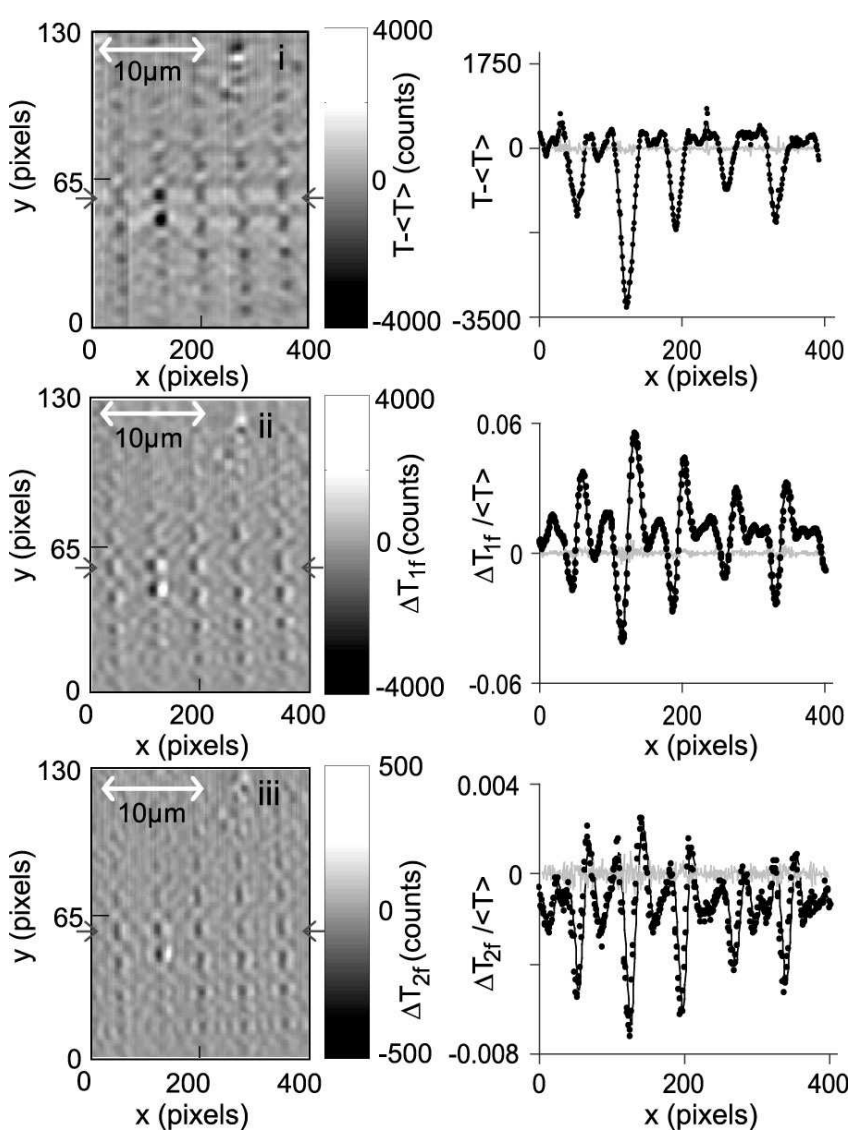

FIG. 3: Spatial modulation microscopy maps of intensity difference from average (i), first harmonic (ii) and second harmonic components (iii) of the spatial modulation signal. Line graphs (I-III) are cross sections of the maps normalized to the average intensity, with (grey lines) noise background after subtraction of moving average.

fitted to a Gaussian beam profile for $T$ and its first and second derivatives for $\Delta T_{1 f}$ and $\Delta T_{2 f}$, as expected from theory [6]. The fits yield a full-width-at-half-maximum of $0.8 \pm 0.1 \mu \mathrm{m}$. This is larger than the diffraction limit due to aberrations in the imaging system. The depth of focus of the SMM signals follows the divergence of the Gaussian beam and results in a decay over $\sim 1.5 \mu \mathrm{m}$. The absence of an out-of-focus background signal is of potential use in imaging of three-dimensional specimens.

Figure 3 shows the SMM image obtained for a $20 \times$ $25 \mu \mathrm{m}^{2}$ area containing nanoantennas. Each line was averaged over 4 frames, resulting in a total acquisition time of 2 seconds. This is an improvement in collection time by more than two orders of magnitude compared to conventional point-scanning SMM [ 6$]$. Only a central 120-pixel part of the 256-pixel array in the y-direction is displayed containing the antenna array. Horizontal crosssections through a row of antennas (indicated by arrows) are shown in Fig. 3(b), where the $1 f$ and $2 f$ components have been normalized to the average intensity $\langle T\rangle$ to obtain the relative spatially modulated transmission. 

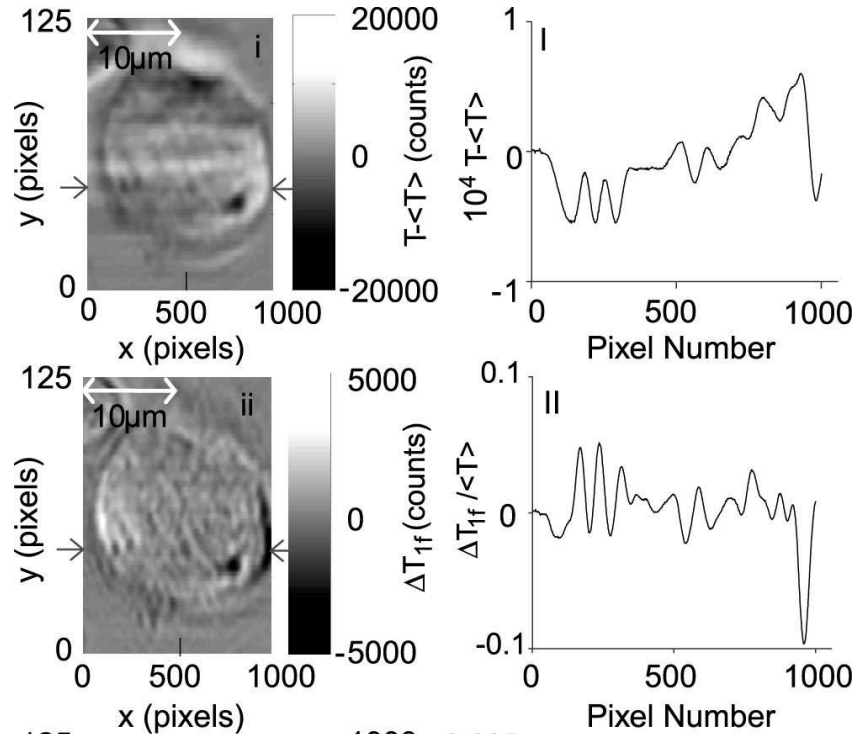

0.1

Pixel Number
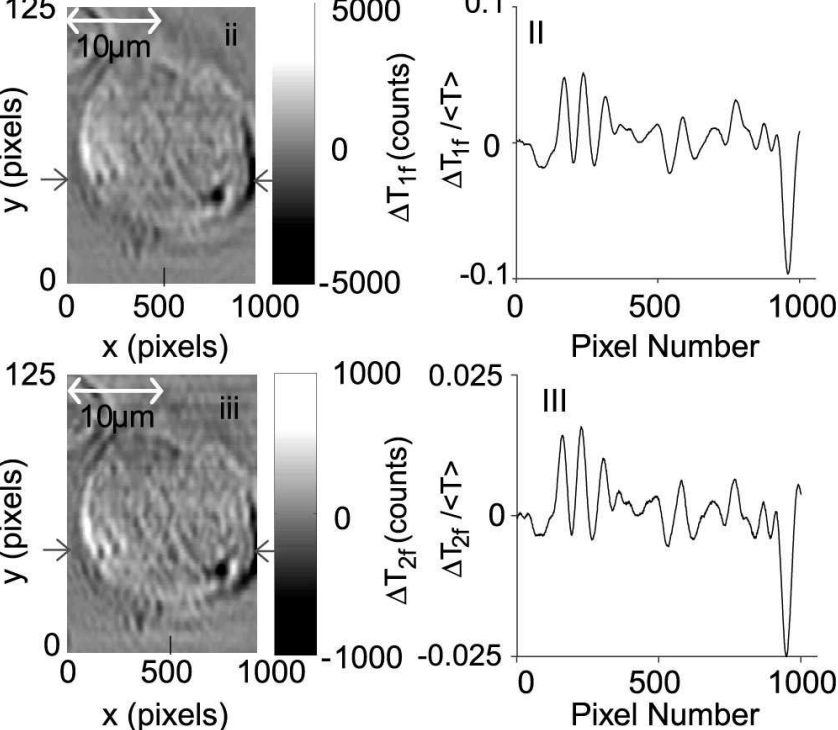

$1000 \quad 0.025$

Pixel Number

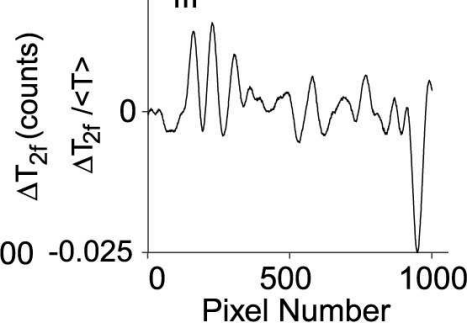

FIG. 4: i - Intensity, ii - 1f and iii - 2f SMM images of dendritic cells along with a cross section.

The noise level of the measurement was calculated by subtracting a 5 point moving average from the original traces taken at a horizontal step size of $50 \mathrm{~nm}$. Resulting noise traces are given by the grey lines in the cross sections of Fig. 3(b). We obtained average noise levels of $3 \times 10^{-4}$ and $1 \times 10^{-4}$ for the $1 f$ and $2 f$ signals respectively. The noise level is exceeds the shot-noise due to the presence of some technical noise in our system at $\mathrm{kHz}$ frequencies. The signal to noise ratio exceeds 100 for the antennas under study at the first harmonic frequency, demonstrating the capability of the system to image individual nanostructures.

In a second experiment, a sample of dendritic cells was incubated with $5 \mu \mathrm{l}$ of a CTAB-coated gold nanorod solution for 2 hours. The gold nanorods, of $16 \times 42 \mathrm{~nm}^{2}$ average dimensions, have a strong optical resonance at around $690 \mathrm{~nm}$ corresponding to a longitudinal surface plasmon resonance. Figure 4 shows an image of a dendritic cell obtained using the intensity, $1 f$ and $2 f$ components of the SMM signal, along with a cross section of each image taken at the position indicated by the arrows. The expected SMM signal from individual nanorods is around $10^{-3}$ [] , i.e. much smaller than the cellular background signals in Fig. 4. The lack of specific contrast thus limits the efficacy of SMM in imaging nanoparticles in cells compared other methods such as photothermal imaging. The $1 f$ and $2 f$ components give the first and second derivatives of the light transmitted through the cell, which results in an enhancement of edges and pointlike objects. This sensitivity of the SMM signal to small variations in optical density in the cell may be of interest as an alternative to phase-sensitive imaging, i.e. phasecontrast or Nomarski, under conditions that phase information is distorted, e.g. for very thick or turbid samples.

In conclusion, a parallel implementation of spatial modulation microscopy has been demonstrated. The theoretical sensitivity of the CMOS demodulation is comparable to state-of-the-art lock-in amplifiers, and the current system allows resolving an array of plasmonic nanoantennas of $200 \mathrm{~nm}$ with a signal-to-noise ratio of 100. This may be improved by reducing sources of classical noise in the system, optimization of the diffractionlimited focus, and averaging over pixels and/or time. Key aspects of SMM are retained by the setup while improving the acquisition time by several orders of magnitude, therefore opening up new applications requiring simultaneous imaging and measurement of multiple nanoparticles e.g. in solid or microfluidic environments. The absence of out-of-focus signals and availability of higherorder derivatives make SMM a potentially useful alternative or addition to phase-contrast techniques.

The authors acknowledge M. Abb for fabrication of nanoantennas and H. Warren for help with cell preparation.

[1] C. Sonnichsen, B. M. Reinhard, J. Liphardt, A. P. Alivisatos, Nat. Biotechnol. 23, 741 (2005).

[2] J. N. Anker, W. P. Hall1, O. Lyandres, N. C. Shah, J. Zhao, R. P. Van Duyne, Nat. Mater. 7, 442 (2008).

[3] M. A. van Dijk, A. L. Tchebotareva, M. Orrit, M. Lippitz, S. Berciaud, D. Lasne, L. Cognet, B. Lounis, Phys. Chem. Chem. Phys. 83486 (2006).

[4] P. Kukura, H. Ewers, C. Müller, A. Renn, A. Helenius, V. Sandoghdar, Nat. Methods 6, 923 (2009).

[5] A. Gaiduk, M. Yorulmaz, P. V. Ruijgrok, M. Orrit, Science 330, 353 (2010).

[6] A. Arbouet, , D. Christofilos, N. Del Fatti, F. Vallée, J. R. Huntzinger, L. Arnaud, P. Billaud, M. Broyer, Phys. Rev. Lett. 93, 127401 (2004)

[7] O. L. Muskens, G. Bachelier, N. Del Fatti, F. Vallée, A. Brioude, X. Jiang, M. Pileni, J. Phys. Chem. C 112, 8917 (2008).

[8] M. Abb, P. Albella, J. Aizpurua, O. L. Muskens, Nano Lett. 11, 2457, (2011).

[9] R. J. Smith, R. A. Light, S. D. Sharples, N. S. Johnston, M. C. Pitter, M. G. Somekh, Rev. Sci. Inst. 81, 024901 (2010).

[10] N.S. Johnston, C. Stewart, R.A. Light, B.R. Hayes-Gill, M.G. Somekh, S.P. Morgan, J.R. Sambles, M.C. Pitter, Electron. Lett. 45, 1090 (2009). 